\title{
DOMINATION SUBDIVISION AND DOMINATION MULTISUBDIVISION NUMBERS OF GRAPHS
}

\author{
Magda Dettlaff, Joanna RaczeK \\ Faculty of Applied Physics and Mathematics \\ Gdańsk University of Technology \\ Narutowicza 11/12, 80-233 Gdańsk, Poland \\ e-mail: magdadettlaff1@pg.edu.pl \\ Joanna.Raczek@pg.gda.pl \\ AND \\ JERZY TOPP \\ Faculty of Mathematics, Physics and Informatics \\ University of Gdańsk \\ Wita Stwosza 57, 80-952 Gdańsk, Poland \\ e-mail: Jerzy.Topp@inf.ug.edu.pl
}

\begin{abstract}
The domination subdivision number $\operatorname{sd}(G)$ of a graph $G$ is the minimum number of edges that must be subdivided (where an edge can be subdivided at most once) in order to increase the domination number of $G$. It has been shown [10] that $\operatorname{sd}(T) \leq 3$ for any tree $T$. We prove that the decision problem of the domination subdivision number is NP-complete even for bipartite graphs. For this reason we define the domination multisubdivision number of a nonempty graph $G$ as a minimum positive integer $k$ such that there exists an edge which must be subdivided $k$ times to increase the domination number of $G$. We show that $\operatorname{msd}(G) \leq 3$ for any graph $G$. The domination subdivision number and the domination multisubdivision number of a graph are incomparable in general, but we show that for trees these two parameters are equal. We also determine the domination multisubdivision number for some classes of graphs.
\end{abstract}

Keywords: domination, domination subdivision number, domination multisubdivision number, trees, computational complexity.

2010 Mathematics Subject Classification: 05C69, 05C05, 05 C99. 
[1] H. Aram, S.M. Sheikholeslami and O. Favaron, Domination subdivision numbers of trees, Discrete Math. 309 (2009) 622-628.

doi:10.1016/j.disc.2007.12.085

[2] D. Avella-Alaminos, M. Dettlaff, M. Lemańska and R. Zuazua, Total domination multisubdivision number of a graph, Discuss. Math. Graph Theory 35 (2015) 315327.

doi:10.7151/dmgt.1798

[3] S. Benecke and C.M. Mynhardt, Trees with domination subdivision number one, Australas. J. Combin. 42 (2008) 201-209.

[4] A. Bhattacharya and G.R. Vijayakumar, Effect of edge-subdivision on vertexdomination in a graph, Discuss. Math. Graph Theory 22 (2002) 335-347. doi:10.7151/dmgt.1179

[5] O. Favaron, T.W. Haynes and S.T. Hedetniemi, Domination subdivision numbers in graphs, Util. Math. 66 (2004) 195-209.

[6] O. Favaron, H. Karami and S.M. Sheikholeslami, Disproof of a conjecture on the subdivision domination number of a graph, Graphs Combin. 24 (2008) 309-312. doi:10.1007/s00373-008-0788-6

[7] M.R. Garey and D.S. Johnson, Computers and Intractability: A Guide to the Theory of NP-Completeness (Freeman, San Francisco, 1979).

[8] T.W. Haynes, S.M. Hedetniemi and S.T. Hedetniemi, Domination and independence subdivision numbers of graphs, Discuss. Math. Graph Theory 20 (2000) 271-280. doi:10.7151/dmgt.1126

[9] T.W. Haynes, S.T. Hedetniemi and P.J. Slater, Fundamentals of Domination in Graphs (Marcel Dekker Inc., New York, 1998).

[10] S. Velammal, Studies in Graph Theory: Covering, Independence, Domination and Related Topics, Ph.D.Thesis (Manonmaniam Sundaranar University, Tirunelveli, 1997).

Received 7 January 2017

Revised 21 August 2017

Accepted 27 November 2017 